\title{
INDUSTRIAL IMPLEMENTATION OF THE NEW NIR LASER-BASED SENSOR FOR MEASURING SURFACE MOISTURE IN POLYMERS
}

\section{Mise en ouvre industrielle d'un nouveau capteur, utilisant le proche infra-rouge laser, pour mesurer l'humidité en surface des polymères}

\author{
Hudoklin $\mathrm{D}^{1, a}$, Muñoz Lopez $\mathrm{I}^{2}$, Begeš $\mathrm{G}^{1}$, Drnovšek $\mathrm{J}^{1}$ and Beguš $\mathrm{S}^{1}$ \\ ${ }^{1}$ University of Ljubljana, Faculty of Electrical Engineering, Laboratory of Metrology and Quality, Trzaska 25, 1000 Ljubljana \\ ${ }^{2}$ Universidad Politécnica de Cartagena (UPCT) Cartagena, Pza. del Cronista Isidoro Valverde, 30202 Cartagena Murcia, Spain
}

\begin{abstract}
In special applications, such as the transient adhesion process over small polymer components used in electric motors, electronic circuit coating or moisture transport in wood; moisture in the upper layers, close to the surface and at the surface, affects the processes more rapidly. Existing measuring principles, such as those based on nuclear magnetic resonance, are cumbersome to use and they are also less suitable for some parts of the moisture range. For this reason, a novel, non-contact, light-reflection type of moisture sensor was investigated, designed and developed (patent pending). It is based on a water molecule's light-absorbing characteristics in the near-infrared (NIR) spectrum. The light from a double laser diode is led through an optical fiber and reflected back from a sample to an InGaAs photodiode detector. The high stability of the relatively low-cost laser source was achieved through a constant-power laser-control circuit. The sensor was designed for measuring the surface and near-surface moisture on a polymer samples with complex geometry and thin walls, which proved to achieve an approximately $0.1 \%$ of effective resolution of bulk moisture content below $2 \%$. The tests showed that the mechanical set-up of the samples plays a significant role. For this reason the special measuring cell was designed in order to be able to use the sensor in an industrial process line, where every year several hundred thousands of polymer samples are manufactured. The advancements from first introduction of the sensor will be presented, together with the experience obtained with its industrial implementation. The later work is in close relation with the activities of the European metrology research programme (EMRP) joint research project Metefnet.
\end{abstract}

\section{Introduction}

Due to a slow moisture transport in most of the materials $[1,2]$, moisture distribution is not uniform during a longer transition period. A resulting moisture profile can have a significantly different effect on some industrial processes [3], such as for instance coating or adhesion, where layers immediately below the surface should have the most influence. In addition, for some samples with complex geometry moisture distribution needs to be measured also at a particular spot and a knowledge of profile in one direction (e.g. in depth) or of a bulk moisture will not suffice. For this reason, a new NIR laser-based sensor was developed for measuring a surface moisture [4]. To obtain focused dimensional targeting the laser is lead through a specially designed optic fiber, which allows also to collect the diffused reflected light. This makes it unique compared to other moisture sensing techniques $[5,6,7,8]$.

On the other hand, targeting a small sample with complex geometry and uneven surface, requires special care to be given to mechanical positioning of the optic fiber. This is especially so, if the sensor is to be used as part of quality control in serial production on-line process, where special focus was given to repeatability and reproducibility of the measurements. The paper will present the mechanical alignment solution, which was tested on a polymer motor parts in the company Domel d.o.o., which is a global developer and supplier of complete electro-motor and component solutions.

\footnotetext{
${ }^{a}$ Corresponding author: domen.hudoklin@fe.uni-lj.si
}

This is an Open Access article distributed under the terms of the Creative Commons Attribution License 4.0, which permits unrestricted use, distribution, and reproduction in any medium, provided the original work is properly cited. 
The sensor development was done in the Laboratory of Metrology and Quality at the University of Ljubljana, Faculty of Electrical Engineering, which is the holder of the national measurement standard for thermodynamic temperature and humidity.

\section{NIR laser-based surface moisture sensor}

\subsection{The measurement principle}

NIR radiation interacting with a sample may be absorbed, transmitted or reflected. As we are interested in the measuring of the surface or near surface moisture in opaque polymer elements we focused on the diffuse reflectance.

Reflectance spectroscopy and Diffuse Reflection Infrared Fourier-Transform (DRIFT) spectroscopy [9, 10] can be used to obtain the infrared spectra of solid materials without any sample preparation. It can provide information about the material's constituents, including the moisture, because characteristic chemical bonds have absorption in the near-infrared (NIR) region. However, these spectra are difficult to interpret, because they depend strongly on the experimental conditions. Others have demonstrated that it is possible to use this principle of on-line measurements without sample preparation and for surface moisture of limited number of applications and materials (e.g. soil, paper). Diffusion, reflectance and transmission properties of radiation propagation in the material are very dependent on the material matrix and geometry of sample. This paper therefore investigates suitability of the proposed sensor by using optic fiber for online measuring of surface and nearsurface moisture in small polymer parts with thin walls and complex geometry.

Radiation aimed at the sample's surface can be directly or internally reflected, absorbed or diffused in all directions. DRIFT spectroscopy utilizes the diffused radiation from the sample and is a result of the radiation beam penetrating into the sample and its diffusion in the sample. The main source of distortion for the DRIFT spectrum is the direct reflection (specular reflection) from the sample surface.

From a theoretical point of view the Lambert-Beer law, is not (directly) applicable for DRIFT spectroscopy. For material samples undergoing preparation to meet the conditions of constant scattering coefficient and infinite sample dilution in a non-absorbing matrix the KubelkaMunk theory could be applied. The Kubelka-Munk model relates sample concentration to the intensity of the measured infrared spectrum [11].

\subsection{Design of the sensor}

The wavelengths of the characteristic absorption bands for water in the near-infrared range are at $0.75 \mu \mathrm{m}, 0.97 \mu \mathrm{m}$, $1.19 \mu \mathrm{m}, 1.45 \mu \mathrm{m}, 1.94 \mu \mathrm{m}$ and $2.95 \mu \mathrm{m}$. Although the range at $1.94 \mu \mathrm{m}$, used, for example in [12] provides a better signal-to-noise ratio, the $1.45 \mu \mathrm{m}$ band was used because of the availability of a compact and relatively low- cost laser-light source and other optical components, e.g., optical fiber and a photodetector.

To determine the moisture content in a sample, two measurements at different wavelengths are necessary: one at the water-absorption band (measurement wavelength) and the other at a wavelength that is considerably less influenced by the moisture of the sample. This reference wavelength was chosen at $1.31 \mu \mathrm{m}$, because of the availability of the compact and relatively low-cost laserlight source. With this wavelength pair a compact, commercial, telecommunications, fiber-optic, laser module could be used, comprising two laser diodes operating at $1490 \mathrm{~nm}$ and $1310 \mathrm{~nm}$, optical isolators and coupling optics.

Due to the small sample size, a specially designed common optical fibers were used to, first, guide the probing NIR radiation to the sample and, secondly, the diffusely reflected radiation back from the sample. This configuration is very convenient for targeting small samples with a non-planar geometry. Additionally, for an on-line measurement in industrial applications, sensitive electronic equipment can, in this way, be separated from a measurement spot.

A block diagram of the moisture sensor system is shown in Fig. 1. The radiation from two lasers is guided via optical fiber to the sample. The dual-wavelength, laserdiode module $(1310 / 1490 \mathrm{~nm})$ with an optic-fiber pigtail is used. The optical output power is $1.47 \mathrm{~mW}$ at $1310 \mathrm{~nm}$ and $1.43 \mathrm{~mW}$ at $1490 \mathrm{~nm}$. Diffusely reflected light from the polymer sample is led through an optic fiber to the photodiode number 1 . The photodiode number 2 with an optic fiber is used to determine the amplitude of measuring and reference light radiating from the optical fiber and is positioned in a way that no reflected light from the sample is collected, see Fig. 1. Due to the low sensitivity of Si photodiodes in this spectral range, InGaAs photodiodes were used instead. The signals from photodiode amplifiers are digitized with an embedded, multichannel, 24-bit, dataacquisition system and processed in real-time using LabView software. The signals used to control the laser diodes are generated by a 16-bit, multichannel system. The length of the data blocks used in data-acquisition is $0.5 \mathrm{~s}$ (10 kHz sampling rate, 5000 samples for one data block), therefore the sample frequency of the sensor is $2 \mathrm{~Hz}$.

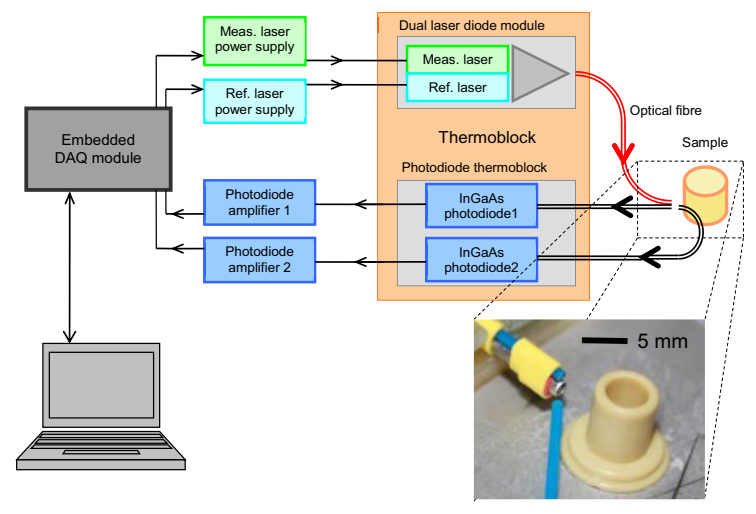

Figure 1. Block diagram of the moisture sensor 


\subsection{Sensitivity improvements}

To be able to achieve the required high sensitivity for this set-up, it was crucial to substantially increase the stability by taking the following steps. Due to the temperature dependence of the laser's optical power, the wavelength and the coupling to the optical fiber, the laser module is mounted on a custom-made, thermoelectrically cooled thermo-block with a temperature controller. Taking into consideration the thermal capacity of the block, the positioning of the regulating temperature sensor and the isolation, the achieved short-term stability was below $1 \mathrm{mK}$, with immunity to ambient temperature change at $1 \mathrm{mK} / \mathrm{K}$. Additionally, a two-channel, laser-diode, power supply was electronically customized in order to drive the laser diodes in a constant optical power mode. This was achieved by adding a feed-back control connection in a laser drive circuitry.

The laser diodes are driven by square-wave-shaped pulses with frequencies of $80 \mathrm{~Hz}$ and $62.5 \mathrm{~Hz}$ for the measurement $(1490 \mathrm{~nm})$ and reference laser $(1310 \mathrm{~nm})$, respectively. These two frequencies are used to avoid any interference from the ambient light $(100 \mathrm{~Hz})$, the power grid $(50 \mathrm{~Hz})$ and so as to have an integer number of periods in the measuring interval $(500 \mathrm{~ms})$, thus avoiding discontinuities at the beginning and at the end of the measurement cycle. The amplitudes are set according to the desired laser power and are adjusted to have the same optical power for both lasers. The necessary current/optical power characteristics were measured for each laser and the appropriate control amplitudes were calculated. By operating both lasers with an AC signal at the same time, the influence of the ambient light and shortterm system drift is reduced. Immunity from the ambient light was established by a demodulating signal with a realtime digital lock-in amplifier, which extracts the amplitude information from the reference and measuring signals simultaneously.

\subsection{Sensor's response}

The tests were performed on a polymer samples, which were kept at different relative humidity levels from $10 \%$, $75 \%$ and $100 \%$, for long enough to allow their bulk moisture level to stabilize. The bulk moisture in the samples was measured by a moisture analyzer. Samples were prepared in two different shapes - disk shape and those that are used in small electric motors, as shown in Figure 1.

To study the difference in sensor's response to a reflected light (surface moisture) and transmitted light (bulk moisture), we carried out the experiment with disc samples made from the same polymer material. The discs had a diameter of $31 \mathrm{~mm}$ and were $0.7 \mathrm{~mm}$ thick. All the discs were initially dried at $60{ }^{\circ} \mathrm{C}$ for 8 hours and weighed. Half of them were then put in a sealed dry container with silicagel at $10 \%$ rh, i.e., dry samples, while the other half of the samples were immersed in water, i.e., wet samples. Before each measurement, each sample was again precisely weighed to be able to calculate the change in the moisture content of the sample over time. After conditioning, the sample discs were stacked by putting different combinations of dry (D) and wet (W) on top of each other. To eliminate the gaps between the individual samples the discs were firmly clamped together to form a sample stack. Prior to clamping, all the individual discs were again weighed to measure the reference moisture uptake. Figure 2 shows the response of the sensor (light reflection) to the different combinations of disc samples in stacks. The combinations are shown above each measurement point, where the letter at the top represents a wet (W) or dry (D) disc (or layer in this case), which is the closest to the source of the sensor's light, i.e., the optic fiber. The letter at the bottom, in contrast, represents the wet or dry disc/layer being on the opposite side of the stack and the farthest away from the sensor. For referencing, an additional dry sample disc was used (marked as REF in Figure 2), which was kept in a dry container $\left(20^{\circ} \mathrm{C}\right.$ and $\left.10 \% \mathrm{rh}\right)$ for a long time. The reference sample's thickness was $2.5 \mathrm{~mm}$, which corresponds to 3 to 4 layers of the above-mentioned stacks.

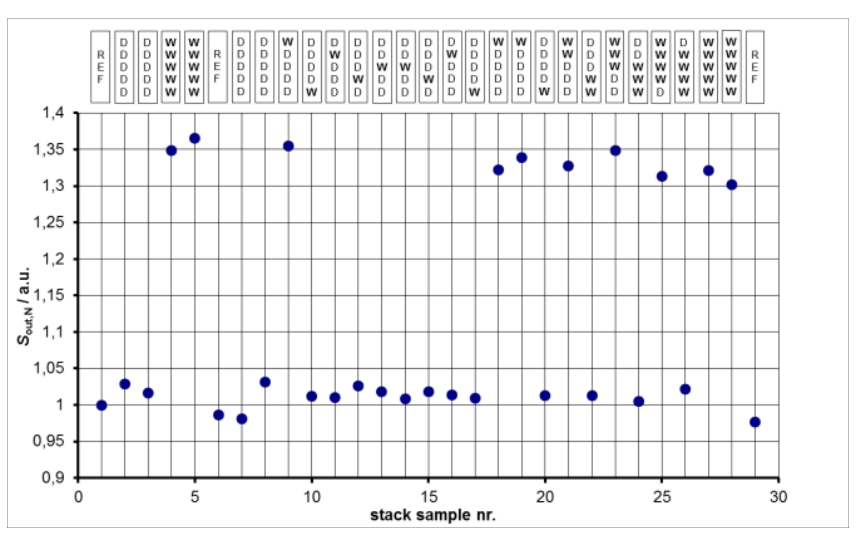

Figure 2. Sensor's output Sout,N to the reflected light for different combination of wet (W) and dry (D) layers/discs in a stack

The results in Figure 2 show that the sensor's signal, which is based on the reflected NIR light, changes significantly only when the first layer/disc of the stack is switched from dry to wet. There is almost no difference, for instance, whether the wet disc is in the second or in any other layer. To make sure that the radiated light from the sensor does penetrate the whole stack and that potential gaps between the layers are not obstructing the path, an additional NIR light detector was also put on the other side of the stack to measure the transmitted light through the stack. The results are shown in Figure 3. 


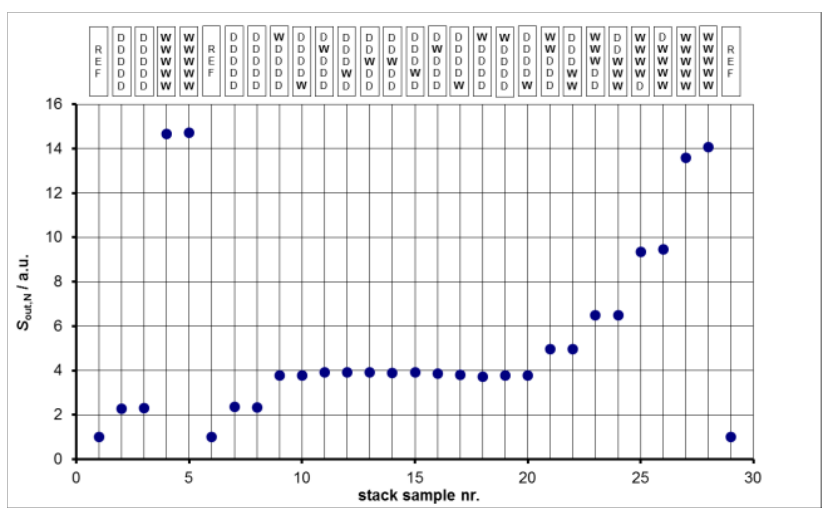

Figure 3. Sensor's output Sout, $N$ of the transmitted light for different combination of wet (W) and dry (D) layers/discs in a stack

The values in both Figure 2 and 3 represent normalized sensor's output Sout, $\mathrm{N}$ in arbitrary units. The results in Figure 3 show that the sensor's response, which is based on transmitted light, depends only on the number of wet layers with consistent steps of $66 \%$ of the signal, when only one wet layer is present (stack sample number 9 to 20 in Figure 3). It does not depend on the location of the wet layer, which was expected. Moreover, it can also be observed that the potential gaps do not have a significant impact on the transmitted light. If more wet layers are present, the step of signal change is increasing exponentially (stack sample number 21 to 28 in Figure 3).

From these observations it can be concluded that the sensor respond to the moisture in the upper layer of the stack, near the surface. The effective depth is seemingly confined to the first layer, which is approximately $0.7 \mathrm{~mm}$ thick. To be able to obtain an even finer resolution, thinner samples would need to be stacked. Because of the limitations of our current technology for making polymer samples, this remains to be further investigated. Moreover, the influence of the gaps could not be totally eliminated, as well as the ratio between the absorbed and the adsorbed moisture. The latter could have an impact on the reflected light directly from the surface and not only from the immediate layers below the surface. The significance of this distinction depends on the application for which the sensor is to be used.

These issues mentioned above will be studied in more detail within the EU EMRP project SIB64 METefnet project devoted to the measurement of moisture in materials, which brings together 10 national-standards laboratories that are active in this field.

\section{Industrial implementation of the sensor}

Sensor was further upgraded to be able to measure surface moisture level in the polymer samples online in the industrial process. The samples are shown in Figure 4.

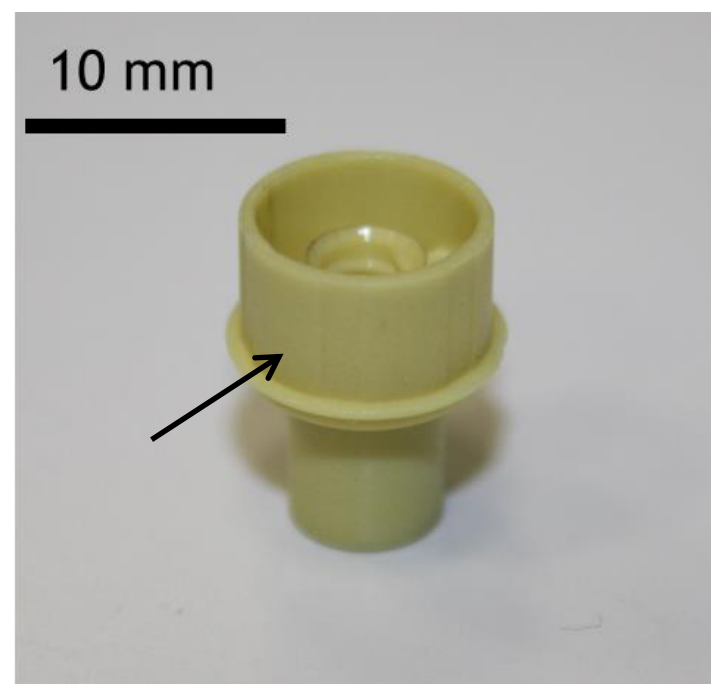

Figure 4. Polymer samples for which

The surface, at which moisture level is to be measured is indicated by an arrow. As it can be seen form the Figure 4, this surface is uneven, as it has expressed valleys. Additionally, the sensor's response is affected also by not completely flat surface on a microscale (speckles) and the angle of the laser beam and also the distance from the optic fiber and the sample, which changes with non-ideal alignment and therefore symmetry of the sample.

For this reason we have designed a special alignment head, which takes into account all these considerations. It is shown in Figure 5.

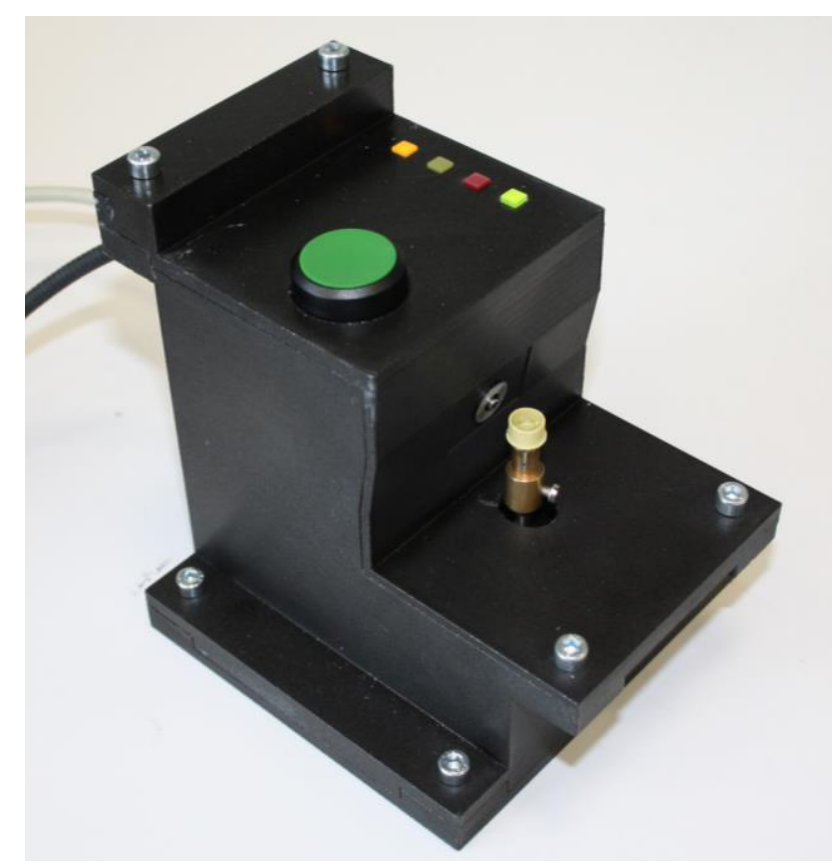

Figure 5. Sample alignment head 
After the sample is inserted, it is fully rotated several times by using micro-stepping stepper motors, while averaging the sensor's output.

The alignment head follows also other process requirements like ergonometry, sample time, dimensional fit-for-purpose etc.

The complete sensor system before the in-preocess installation is shown in figure 6. It comprises of the main sensor part with embedded electronics on the left, alignment head on the right and the black optic fiber in between.

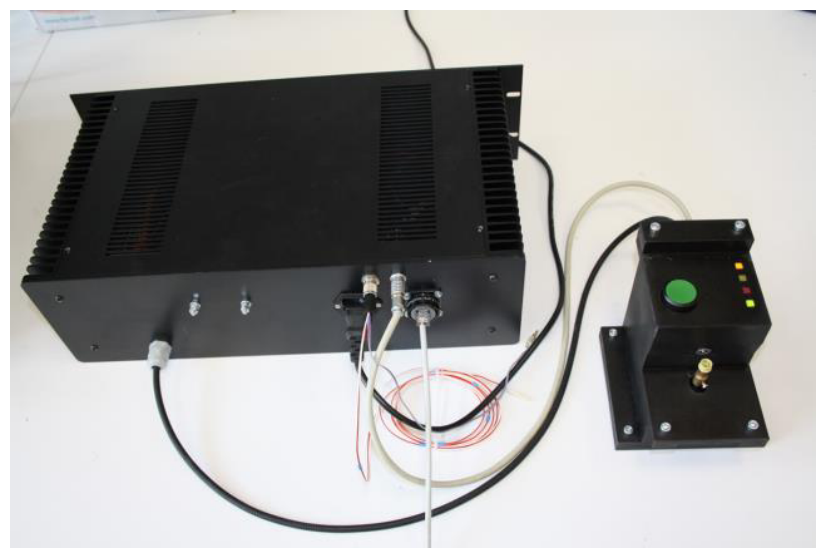

Figure 5. The complete system for online measuring of the surface moisture for polymer samples

\section{Summary}

The paper summarizes the new system for measuring surface moisture in polymer sample with complex geometry. It is based on NIR laser technology using DRIFT spectroscopy with specially configured optical part.

This sensor was further adapted, so that it would be able to be used in industry for the purpose of quality control of adhesion process. Having constructed a special alignment head, the results show a high level of repeatability.

Further developments will be focused on the development of the calibration system, with which such surface moisture sensors could be calibrated. The work is already in progress in the scope of joint research project SIB64 Metefnet of the European metrology research programme (EMRP).

\section{Acknowledgements}

This work is partially funded by the EMPIR initiative that is co-funded by the European Union's Horizon 2020 research and innovation programme and the EMPIR Participating States.

\section{References}

[1] H. Abe in K. T. Yamadab, ,Performance evaluation of a trace-moisture analyzer based on cavity ringdown spectroscopy: Direct comparison with the NMIJ trace-moisture standard," Sensors and Actuators A: Physical, Izv. 165, pp. 230-238, 2011.

[2] B. I. Choi, H. Nham, S. B. Woo, J. C. Kim in S. Y. Kwon, „Design, Construction and Performance Test of a Reflection Type Quadruple Beam Infrared Moisture Meter," v Proc. of 5th International Symposium on Humidity and Moisture - ISHM 2006, Rio de Janeiro, Brasil, 2006.

[3] R. Benyon, S. A. Bell, N. Boese in M. Heinonen, „A roadmap for humidity and moisture measurement,“ Int. J. Thermophys, Izv. 29, št. 5, pp. 1537-1543, 102008.

[4] S. Beguš, G. Begeš, J. Drnovšek in D. Hudoklin, „A novel NIR laser-based sensor for measuring the surface moisture inpolymers," Sensors and Actuators A: Physical, Izv. 221, pp. 53-59, 2015.

[5] K. Saarinen in P. Heino, „Moisture effects on adhesion of non-conductive adhesive attachments," Soldering \& Surface Mount Technology, Izv. 22, št. 1, p. 41-46, 2010.

[6] R. Cuccaro, R. M. Gavioso, G. Benedetto, D. Madonna Ripa, V. Fernicola in C. Guianvarc'h, „Microwave Determination of Water Mole Fraction in Humid Gas Mixtures, “International Journal of Thermophysics, Izv. 33, št. 8-9, pp. 1352-1362, 2012.

[7] H. Huang, Y. Haiyan in Y. Yibin, „Near infrared spectroscopy for on/in-line monitoring of quality in foods and beverages: A review, "Journal of Food Engineering, Izv. 87, št. 3, pp. 303-313, 2008.

[8] F. Casanova, J. Perlo in B. Bluemich, „Depth profiling by Single-Sided NMR, " v NMR Imaging in Chemical Engineering, Weinheim, Wiley-VCH, 2006, pp. 107-123.

[9] B. Guo, Q. Zou, Y. Lei in D. Jia, ,Structure and Performance of Polyamide 6/Halloysite Nanotubes Nanocomposites,“ Polymer Journal, Izv. 41, št. 10, pp. 835-842, 2009.

[10] M. P. Fuller in P. R. Griffiths, „Diffuse Reflectance Measurements by Infrared Fourier Transform Spectroscopy,“'Analytical Chemistry, Izv. 50, št. 13, pp. 1906-1910, 1978.

[11] P. Kubelka in F. Munk, ,Ein Beitrag zur Optik der Farbanstriche,“'Z.Tech. Phys., Izv. 12, p. 593-601, 1931.

[12] B. I. Choi, S. B. Woo in J. C. Kim, „Measurement of ultra-low moisture permeation through epoxy sealing in OLED encapsulation, "v XX IMEKO World Congress Metrology for Green Growth, Busan, Republic of Korea, 2012. 